\title{
Distributed Compression for Condition Monitoring of Wind Farms
}

\author{
Vladimir Stanković, Lina Stanković, Shuang Wang, and Samuel Cheng
}

\begin{abstract}
A good understanding of individual and collective wind farm operation is necessary for improving the overall performance of the wind farm 'grid', as well as estimating in real time the amount of energy that can be generated for effectively managing demand and supply over the smart grid. This paper proposes a scheme for compressing wind speed measurements exploiting both temporal and spatial correlation between the readings via distributed source coding. The proposed scheme relies on a correlation model based on true measurements. Two compression schemes are proposed, both of low encoding complexity, as well as a particle-filtering based belief propagation decoder that adaptively estimates the nonstationary noise of the correlation model. Simulation results using realistic models show significant performance improvements compared to the scheme that does not dynamically refine correlation.
\end{abstract}

Index Terms-Wind Farms, Distributed Compression, Distributed Source Coding, Adaptive Decoding

\section{INTRODUCTION}

The proliferation of alternative renewable sources of energy urges the development of informed decision making and communications technologies that will maximize sustainable energy generation but still provide uninterrupted service through efficient power distribution planning. The key to the provision of efficient planning of transmission power systems is availability of reliable information on the available sources of sustainable energy at any time.

For example, wind energy as one of the most popular sources of renewable energy depends on temporal wind speed, which varies in time and space. To enable power distribution system planning, precise and timely information of current wind speed (including direction and orientation) is required. This information must be conveyed in near real time to a central substation from each of the wind farms that supplies particular region. After collecting the data from all wind farms, the central substation can estimate temporal wind energy generation. There have been studies in the literature on modeling wind speed, but to the best of our knowledge, nothing has been reported on effective communications of the readings exploiting correlation between wind turbine measurements. Communication of wind measurements is especially important since the changes in wind speed can significantly reduce the amount of generated renewable energy and consequently affect supply and demand. Indeed, designing an effective power

Vladimir Stanković and Lina Stanković are with the Dept of Electronic and Electrical Engineering, University of Strathclyde, Glasgow, UK. Tel: +44-(141)-5482679. Shuang Wang and Samuel Cheng are with School of Electrical and Computer Engineering, University of Oklahoma, Tulsa, OK 74135-2512, USA. E-mail:\{vladimir, lina.\}stankovic@eee.strath.ac.uk, $\{$ shuangwang, samuel.cheng\}@ou.edu. network infrastructure will depend on measurements made to ensure the export of power from areas where wind generation is high due to high wind speeds and import of power where the speeds are low. To make these decisions in a timely manner, measured wind readings from all neighboring (e.g., national) wind farms need to be delivered and processed continuously [1], [2].

Since several studies have shown high wind speed variations during a single day, it is important to accurately transmit timely wind parameters to the central substation, for immediate generation assessment and future predictions. This paper focuses on effectively communicating wind speed information from multiple wind farms to the central substation. The main communication challenges are related to the fact that the wind farms are energy-constrained, remote and wireless transmission channels are bandwidth limited. This calls for efficient low-complexity compression mechanisms that must operate in real time. Moreover, due to the proximity of neighboring wind farms, the proposed compression techniques should capture spatial correlation (besides temporal) of the signals measured without requiring communications between the turbines. As demonstrated in [1], [2], there is a huge correlation between wind measurements in neighboring wind farms (e.g., the study in [2] provided models of correlation between 14 wind farms in the UK).

We resolve the above challenges through the use of distributed compression, or distributed source coding (DSC). DSC is an information-theoretical concept introduced in [3], [4], [5] that refers to separate compression and joint decompression of mutually correlated sources. DSC is especially suitable for the targeted scenario because it provides very low complexity encoding solutions, and enables exploiting spatial correlation without the need for data exchange between encoding nodes. DSC has been proposed for data gathering in wireless sensor networks where multiple, closely related sensors are expected to have statistically dependent readings. Targeting wireless sensor networks and video surveillance networks, a practical DSC code design using channel coding was proposed in 1999 under acronym DISCUS [6]. DISCUS was followed by many improved solutions that exploit more advanced channel codes, provide rate allocation flexibility, decrease complexity, or enable low channel codeword sizes (see [7], [8] for a review).

DSC is particularly suitable for the wind turbine measurement compression due to resource limited encoding at the turbine and high coding efficiency due to exploitation of spatial correlation among turbine sites. Indeed, the effective DSC design can capture both temporal correlation 
between successive readings at a single turbine site and spatial correlation between the readings of closely located sites. However, a prerequisite for efficient DSC code design is accurate estimation of the correlation between the measured data. Indeed, a usual assumption in the design of distributed compression codes is that all encoders and the decoder have perfect knowledge of the statistics of the measured data, i.e., the correlation noise among sources. However, often the correlation model is unknown, or the statistics of the sources vary over time. Indeed, in the targeted wind farm scenario, we are dealing with compression of non-stationary sources whose intensity and direction changes irregularly. This poses a huge challenge on the underlying compression scheme since correlation unpredictably varies over time.

Recently, in [9], particle filtering was integrated into the DSC decoding process to estimate and track correlation changes over time. The scheme maintains low encoding complexity, and introduces changes to conventional DSC coding only at the decoder side. The scheme is used in [10] to track correlation changes in high-motion video within a distributed video coding (DVC) design [11], [12]. The concept has been successfully extended to multiview images captured from solar system satellites in [13].

In [1], using a vector autoregression (VAR) approach, a spatio-temporal correlation model between wind speed readings in neighbouring wind turbines is proposed based on true measurements in Scotland. The model captures both the time varying nature of the wind speed at each site as well as the spatial correlation between the readings at neighboring sites. The additive correlation noise is in general nonstationary which is an additional challenge for the code design calling for effective correlation tracking.

In this paper we apply the information-theoretical concepts of [9], [10] to distributed compression of wind speed information from wind farms. The proposed multiterminal source coding scheme is of low encoding complexity, but still able to effectively exploit spatio-temporal correlation within measured data. The wind farms compress their measurements using DSC and send the compressed data to the central substation, where the joint decoder estimates and tracks the change of correlation using particle-filtering based belief propagation. The scheme can be integrated in the IEC 61400-25 international standard for monitoring of wind power plants and control information exchange.

The paper is organized as follows. Section II gives a brief overview of DSC. Section III describes the system model and the proposed compression scheme including a brief overview of the decoding process. Simulation results of our proposed DSC setups are presented in Section IV. Section V concludes the paper and outlines future work.

\section{Distributed SOURCE CODING (DSC)}

DSC considers independent compression of correlated data, where correlation is exploited at the decoder side. Let $X$ and $Y$ be two correlated sources that should be compressed and sent to a central point for decoding. The compression must be done independently, that is, $X$ and $Y$ do not communicate, whereas decompression is joint. For discrete $X$ and $Y$ and lossless compression, in 1973, Slepian and Wolf [3] showed, surprisingly, that asymptotically, separate compression can be as efficient as joint compression, as long as $X$ and $Y$ are decompressed jointly. The Slepian-Wolf setup is shown in Figure 1, where $R_{X}$ and $R_{Y}$ are the respective compression rates of encoded sources $X$ and $Y$. The DSC problem was introduced in [3] and was followed by intense informationtheoretical research into developing theoretical compression bounds and quantifying coding gains for similar setups.

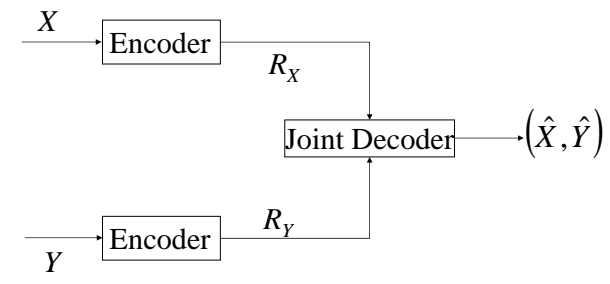

Fig. 1. Slepian-Wolf setup with two sources.

There are two types of Slepian-Wolf coding setups investigated in the literature [14]. The first one, presented in Figure 1 , is called non-asymmetric Slepian-Wolf coding, where both sources need to compress their information exploiting correlation. In the second setup, called asymmetric Slepian-Wolf (SW) coding or source coding with decoder side information, one source, say $X$, needs to be compressed assuming that another source, $Y$, called side information, is available, uncompressed, at the decoder as side information.

An interesting extension of Slepian and Wolf's result is reported in 1976 by Wyner and Ziv [4] who considered a lossy version of the asymmetric SW coding. In the WynerZiv (WZ) setup, $X$ needs to be recovered under a certain distortion constraint and can be discrete or analogue. Wyner and Ziv showed that if $X$ and $Y$ are jointly Gaussian, then under a mean square error (MSE) distortion constraint, there is no rate loss due to the absence of $Y$ at the encoder. For other correlation models, there is a limited coding loss, which is usually small [15].

The extension of general, non-asymmetric SW coding to the lossy case is referred to as multiterminal source coding [5]. There are two types of multiterminal (MT) source coding schemes. One, called direct MT source coding, where two correlated sources need to be compressed independently and recovered jointly under distortion criteria. Another type is indirect MT source coding, where a single source is observed by two (or more) sensors, where each sensor observes only a noisy version of the sources. The sensors need to compress their observations and transmit them to the central unit, which recovers the source using a single distortion measure. In this paper we focus on direct MT source coding. 
Motivated by the need for distributed compression in wireless sensor networks, in 1999, the first practical DSC scheme, or more precisely WZ coding scheme, based on channel codes appeared [6]. It was followed by many improved and more flexible solutions (see [7], [8] for reviews). Practical code designs for non-asymmetric SW coding and direct and indirect MT source coding are reported in [14], [16] and references therein. Nowadays, DSC designs are at the core of distributed video coding (DVC), a novel paradigm that reduces the complexity of video encoding by shifting motion estimation complexity to the decoder side [11], [12] and opening new possibilities of video distribution.

The main assumption in the proof of SW and WZ theorems and in the majority of developed code designs, is the knowledge of statistics at the encoder and the decoder. That is, both encoder and decoder must know correlation between $X$ and $Y$ before coding takes place. In many cases this is an unrealistic assumption since correlation varies over time. In [9], a WZ coding scheme is proposed that unifies the process of online temporal correlation estimation and SW decoding into a single iterative process providing better statistics estimate and consequently improved performance. The correlation model, assumed in [9], is based on simple additive white Gaussian noise. The contributions of this paper are: (i) tracking of both spatial and temporal correlation statistics, (ii) a direct MT source codec design, (iii) a decoder based on particlebased belief propagation, whose algorithm operates on a factor graph where the correlation noise is modelled as [1], (iv) performance bounds of the proposed direct MT source coding scheme.

\section{THE PROPOSED SCHEME}

In this section we describe the wind farm system and the correlation model used to model wind speed. This is followed by a description of the proposed solution.

\section{A. System setup}

The targeted scenario is shown in Figure 2, where the measurements from each wind farm are first quantized, then independently SW-encoded for compression and possibly entropy coded for compression of side information that will be generated at the decoder. Both SW-encoded and entropy coded measurements are sent to the central substation for decoding. That is, closely located wind farms are measuring wind speed, compressing the readings and sending the compressed data to the central substation for decoding. It is assumed that the relative position of the wind farms is known and that their compression units are synchronized. For simplicity, we consider the case of two farms only. In the case of multiple farms, to keep complexity low, one would group them in pairs and independently perform coding on each pair.

Let $X_{1}(t)$ and $X_{2}(t)$ be the wind speed measured at wind farm 1 and 2 , respectively, at time $t$. Let $\mathbf{X}(t)=$ $\left[X_{1}(t) X_{2}(t)\right]^{T}$. It was shown in [1] that a good model relating the measurements in the two wind farms is given by:

$$
\mathbf{X}(t)=\boldsymbol{\Phi}_{\mathbf{1}} \mathbf{X}(t-1)+\boldsymbol{\Phi}_{\mathbf{2}} \mathbf{X}(t-2)+\mathbf{n}(t),
$$

where $\mathbf{n}(t)=\left[n_{1}(t) n_{2}(t)\right]^{T}$ is white noise with $n_{1}(t)$ and $n_{2}(t)$ being nonstationary noises at wind farms 1 and 2, respectively; $\boldsymbol{\Phi}_{i}, i=1,2$ is a matrix that depends on the relative position of the farms and is known at the encoder and decoder.

As suggested in [2] based on measured data in $14 \mathrm{UK}$ wind farms, elements of $\boldsymbol{\Phi}$ can be identified by multivariate least squares estimation, and reflect the influence of one site to another.

Each wind farm needs to compress its readings and send the data to the central substation, which collects the data from both farms before attempting to jointly decompress them. We assume that communication is always error free (via effective physical-layer protection) and focus on distributed compression next. For simplicity in the following, we assume that $\boldsymbol{\Phi}_{\mathbf{2}}$ is a zero matrix, and attempt to exploit only first-order correlation dependency between the two random variables, that is, we simplify (1) to:

$$
\mathbf{X}(t)=\mathbf{\Phi} \mathbf{X}(t-1)+\mathbf{n}(t) .
$$

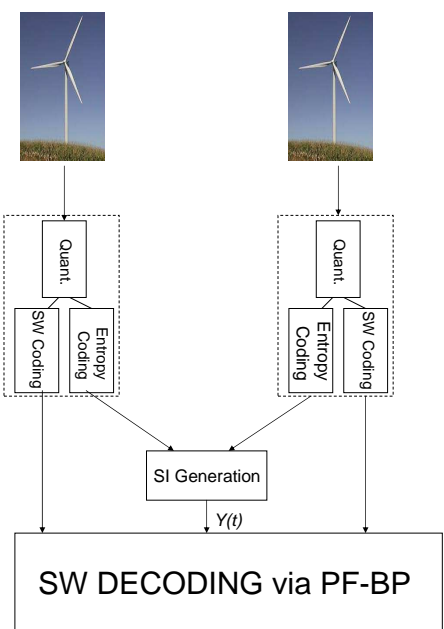

Fig. 2. The block diagram of the proposed system.

\section{B. The proposed solution}

In this section, we propose two coding solutions: one which we call the asymmetric scheme and another, the symmetric scheme, which rely on direct MT source coding [5], [16].

1) Asymmetric scheme: The block diagram of the proposed asymmetric scheme is shown in Figure 3. In this scheme, one farm, say farm 2, sends its measured data $X_{2}(t)$ using quantization (Q) possibly followed by conventional entropy coding, such as Huffman coding. After decoding, the reconstructed measurement $\hat{X}_{2}$ is used at the decoder to generate side information $Y(t)$ for $\mathrm{WZ}$ decoding of compressed readings from the first wind farm. The contents of $\boldsymbol{\Phi}$ can be seen as representing the fractional contributions from $X_{1}(t-1)$ and 
$X_{2}(t-1)$ towards $X_{1}(t)$, and similarly for $X_{2}(t)$, that is:

$$
X_{1}(t)=\phi_{11} X_{1}(t-1)+\phi_{12} X_{2}(t-1)+n_{1}(t),
$$

and

$$
X_{2}(t)=\phi_{21} X_{1}(t-1)+\phi_{22} X_{2}(t-1)+n_{2}(t),
$$

where $\phi_{i j}$ is the element in matrix $\boldsymbol{\Phi}$ in (2) in the $i$-th row and $j$-th column. Thus, $\boldsymbol{\Phi}$ provides information about spatial and temporal correlation between wind speed measurements.

The encoder in farm 1, splits the measurements into odd $\left(X_{1}(2 \tau-1)\right)$ and even $\left(X_{1}(2 \tau)\right)$, where $\tau$ is a positive integer in the rest of the paper. The odd measurements are multiplied by $\phi_{21}$. Both $\phi_{21} X_{1}(2 \tau-1)$ and $X_{1}(2 \tau)$ are quantized sampleby-sample, using a scalar quantizer $(\mathrm{Q})$. Then, quantization indices are split into bitplanes and each bitplane is independently compressed using SW coding via low density parity check (LDPC) codes. This way two syndrome vectors are formed $S_{1}(2 \tau-1)$ and $S_{1}(2 \tau)$ for odd and even measurements, respectively, for each set of $N$ measurements. Note that multiplication by $\phi_{21}$ is done to avoid magnification of the noise at the decoder. Bitplane-by-bitplane compression enables more flexible compression rate selection since different bitplanes will be correlated in different ways, e.g., the most significant bitplanes (MSB) are correlated the most, and so forth.

The decoder generates side information as:

$$
Y(2 \tau-1)=\hat{X}_{2}(2 \tau)-\phi_{22} \hat{X}_{2}(2 \tau-1),
$$

where $\hat{X}_{2}(t)$ is the estimated measurement of farm 2 at time $t$.

The WZ decoder uses $Y(2 \tau-1)$ as side information and received syndromes for odd measurements $S_{1}(2 \tau-1)$ to recover $\hat{X}_{1}(2 \tau-1)$. That is, the decoder uses the correlation channel model [7], [14]:

$$
Y(2 \tau-1) \approx \phi_{21} X_{1}(2 \tau-1)+n_{2}(2 \tau),
$$

that follows from (5) and (4), to estimate $X_{1}(2 \tau-1)$ as $\hat{X}_{1}(2 \tau-1)$. Note that this is done at time $2 \tau$ (since it depends on $\hat{X}_{2}(2 \tau)$ ), thus $X_{1}(2 \tau-1)$ is decoded introducing a delay of one time sample. The sample at time $2 \tau$ is then decoded from

$$
\hat{X}_{1}(2 \tau)=\phi_{11} \hat{X}_{1}(2 \tau-1)+\phi_{12} \hat{X}_{2}(2 \tau-1)+n_{1}(2 \tau),
$$

using

$$
Y(2 \tau)=\phi_{11} \hat{X}_{1}(2 \tau-1)+\phi_{12} \hat{X}_{2}(2 \tau-1),
$$

as side information and the received syndrome $S_{1}(2 \tau)$ that corresponds to the even measurements of farm 1 .

Note that the decoding is done in two stages: during the first stage spatial correlation is exploited while in the second, temporal correlation.

The odd and even samples can be sent at different rates and thus can employ different SW codes. Alternatively, farm 1 can compress the difference $X_{1}(2 \tau)-\phi_{11} X_{1}(2 \tau-1)$ using $\mathrm{SW}$ coding and then the decompression with side information $\phi_{12} \hat{X}_{2}(2 \tau-1)$ can proceed in parallel with the decompression of $X_{1}(2 \tau-1)$ with side information $Y(2 \tau-1)$.

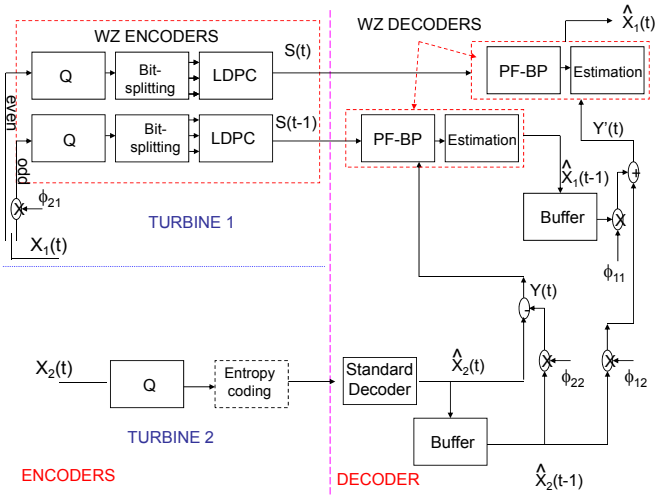

Fig. 3. The proposed asymmetric scheme.

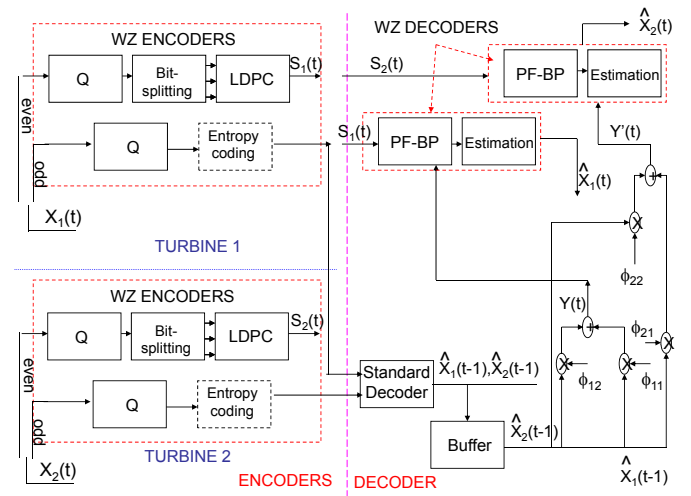

Fig. 4. The proposed symmetric scheme.

2) Symmetric scheme: In the asymmetric scheme described above, in one time window only one farm uses DSC, whereas the other one resorts to conventional compression. Using time-sharing or channel code partitioning (see [14], [16] and references therein), it is possible to balance the DSC rate load of the the wind farms. Another way to evenly distributed DSC compression is the proposed symmetric scheme shown in Figure 4.

Both farms conventionally compress all odd measurements $\left(X_{1}(2 \tau-1), X_{2}(2 \tau-1)\right)$ using scalar quantization followed by entropy coding. All even measurements $\left(X_{1}(2 \tau), X_{2}(2 \tau)\right)$ at both farms are compressed using DSC with scalar quantization followed by bitplane-by-bitplane LDPC encoding for syndrome forming as in the asymmetric scheme.

The decoder first recovers odd measurements from both farms as $\hat{X}_{1}(2 \tau-1)$ and $\hat{X}_{2}(2 \tau-1)$. Then, to recover $X_{1}(2 \tau)$, the decoder employs SW decoding assuming:

$$
\hat{X}_{1}(2 \tau)=\phi_{11} \hat{X}_{1}(2 \tau-1)+\phi_{12} \hat{X}_{2}(2 \tau-1)+n_{1}(2 \tau),
$$

using $Y_{1}(\tau)=\phi_{11} \hat{X}_{1}(2 \tau-1)+\phi_{12} \hat{X}_{2}(2 \tau-1)$ as side information.

Then, $X_{2}(2 \tau)$ is recovered as:

$$
\hat{X}_{2}(2 \tau)=\phi_{21} \hat{X}_{1}(2 \tau-1)+\phi_{22} \hat{X}_{2}(2 \tau-1)+n_{1}(2 \tau),
$$

using $Y_{2}(\tau)=\phi_{21} \hat{X}_{1}(2 \tau-1)+\phi_{22} \hat{X}_{2}(2 \tau-1)$ as side information. 
Note that side information captures both spatial and temporal correlation. As in the case of asymmetric scheme, two SW decodings are necessary, but in contrast to the asymmetric scheme where these two decodings must be done serially, in the symmetric scheme they can be performed in parallel. Note that both proposed scheme essentially employ asymmetric direct MT source coding.

3) Rate-distortion Analysis: Next, we estimate the required compression rate assuming two zero-mean Gaussian memoryless sources, ideal quantization, and use the mean squared error (MSE) distortion metric. First we look at the asymmetric scheme.

Let the required rate to compress measurements of farm 1 to achieve MSE $D_{1}$ be:

$$
R_{X_{1}}\left(D_{1}\right)=R_{\text {odd } 1}\left(D_{1}\right)+R_{\text {even } 1}\left(D_{1}\right)
$$

where $R_{o d d 1}\left(D_{1}\right)$ and $R_{\text {even } 1}\left(D_{1}\right)$ are the required rates for odd and even measurements, respectively.

Note that the correlation channel is a Gaussian channel given by (6), where the side information $Y(2 \tau-1)$ is given by (5). Assuming that $n_{2}(2 \tau)$ is a Gaussian memoryless source independent of $X_{1}(2 \tau-1)$, then $R_{o d d 1}\left(D_{1}\right)$ is:

$$
R_{o d d 1}\left(D_{1}\right)=\frac{1}{2} \log +\frac{\sigma_{N_{2}}^{2}}{D_{1}\left(1+\frac{\sigma_{N_{2}}^{2}}{\left(\phi_{21} \sigma_{1}\right)^{2}}\right)},
$$

where $\sigma_{N_{2}}^{2}$ is variance of $n_{2}(t)$ and $\sigma_{1}^{2}$ is variance of $X_{1}(t)$. Here $\log ^{+} p=\log p$ for $p>0$ or 0 , otherwise. For derivation, see [7] and references therein.

From the second correlation channel (7) and side information (8), we get:

$$
R_{\text {even } 1}\left(D_{1}\right)=\frac{1}{2} \log ^{+}\left(\frac{\sigma_{N_{1}}^{2}}{D_{1}}\right) .
$$

Then from (11), (12), and (13) the minimum rate need to compress wind farm 1 measurements given farm 2 measurements at MSE level $D_{1}$ is:

$$
R_{X_{1}}\left(D_{1}\right)=\log ^{+}\left(\frac{\phi_{21} \sigma_{N_{1}} \sigma_{N_{2}} \sigma_{1}}{D_{1} \sqrt{\phi_{21}^{2} \sigma_{1}^{2}+\sigma_{N_{2}}^{2}}}\right) .
$$

The rate-distortion characteristic of farm 2 is given by:

$$
R_{X_{2}}\left(D_{2}\right) \approx \frac{1}{2} \log ^{+} \frac{\sigma_{X_{2}}^{2}}{D_{2}}
$$

where $\sigma_{X_{2}}^{2}$ is the variance of $X_{2}(t)$ (see [7] and references therein).

It can easily be confirmed that both asymmetric scheme and symmetric scheme have the same theoretical performance, that is, the same total rate sum $R=R_{X_{1}}+R_{X_{2}}$. Thus, the individual rate limits of the wind farms for the symmetric scheme are $R_{X_{1}}(D)=R_{X_{2}}(D)=R / 2$, where $R$ is the sum of the righthand side of (14) and (15) with appropriate $D$. Note, however, that in the practical realization of the symmetric scheme, there is no error propagation due to successive SW decoding.
4) Adaptive Decoding: The decoding procedure is an adapted version of [9] and [10], where a DVC application with different correlation noise model and side information generation is tackled. The compressed stream (syndromes) is sent to a graph-based SW decoder, which uses the belief propagation (BP) algorithm with particle filtering $(\mathrm{PF})$ to estimate current correlation noise $n_{2}(t)$ and decompress the source. The PFBP-based algorithm operates on a joint 3D factor graph that represents the probabilistic relationship among SW coding, bitplane coding and correlation tracking - see Figure 5 and [10] for details of the factor graph construction and the PF-BP algorithm. These are mapped into appropriate variables nodes and factor nodes, where variables nodes denote unknown variables such as SW coded bits and correlation variance and factor nodes represent the algebraic connection among multiple variable nodes. In this paper, correlation variable nodes are modelled as Gaussian and vary slowly over time.

In the PF-BP algorithm, messages are passed iteratively between connected variable nodes and factor nodes in the different regions of the graph (region 1: bipartite graph connecting correlation variable and factor nodes, region 2: 2D SW factor-sub-graph representing SW code used for each bitplane) until the algorithm converges or until a fixed number of iterations is reached. These messages (inferences or beliefs on source bits and correlation) will represent the influence that one variable has on another. Standard BP (the sum-product algorithm), generally used for SW LDPC decoding, can handle only discrete variables. The correlation variance, however, is not a discrete variable, since it varies continuously over time. We therefore resort to PF, which is integrated within the standard BP algorithm in order to handle continuous variables.

PF estimates the a posteriori probability distribution of the correlation variable node by sampling a list of random particles with associated weights. Systematic resampling is applied once all weights have been updated for all particles in each variable node to discard particles with negligible weight and concentrate on particles with larger weights. To maintain diversity of the particles, the new particle locations are perturbed by applying the random walk Metropolis-Hastings algorithm. The weight of each particle is then reset to a uniform weight for each particle. A new codeword is generated at the end of each iteration until the BP algorithm finds a valid codeword or until it reaches a maximum number of iterations.

\section{Simulation Results}

In this section we report results of our simulations for the case of two neighboring wind farms measuring wind speed and sending their compressed readings to the central substation. We show the results as coding/compression rate required for DSC versus MSE between the original samples and reconstructed ones. All results are averaged over 100 simulations, and MSE contains both "fine" quantization distortion as well as "coarse" distortion due to SW decoding errors [7]. In all our experiments we set matrix $\Phi$ to contain all 0.5 , which puts equal weight on spatial and temporal signal component (see (3)-(4)). The code length is always 10,000 symbols/samples, and each sample is quantized into $q$ bits. 


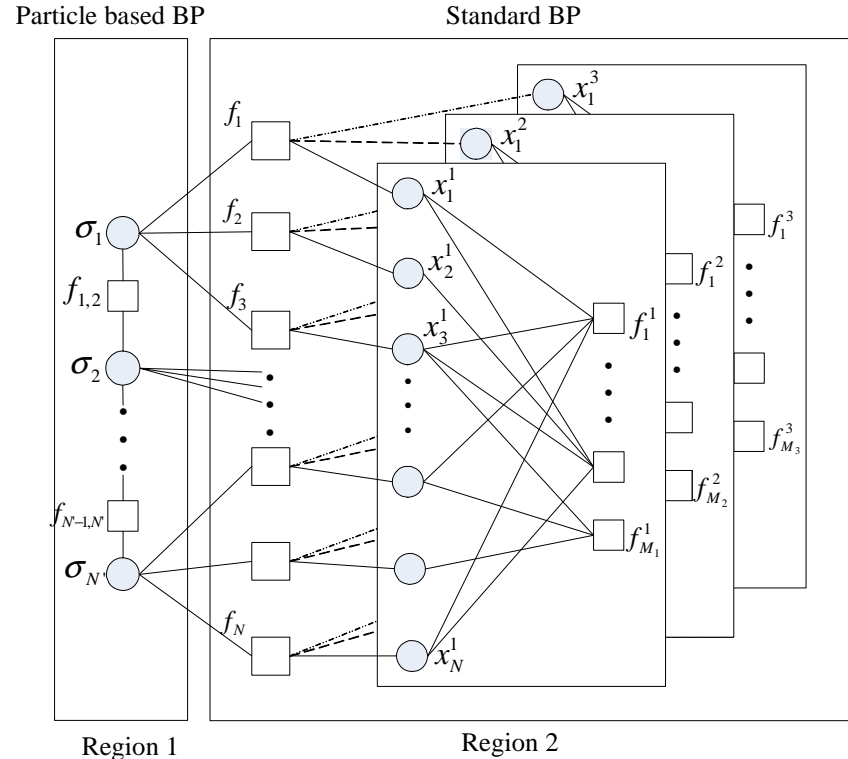

Fig. 5. Factor graph for the PF-BP-based adaptive decoder.

We present our results for the symmetric setup only, which slightly outperforms the asymmetric setup, because of error propagation in the latter. Note that in the symmetric setup, the employed channel code length is 5000 bits for each bitplane since every second measurement is compressed using DSC by each wind farm.

The number of particles tracking each correlation variable is set to 10 and the variance of the correlation between particles is $\sigma_{\gamma}^{2}=0.07$. These values were heuristically found to provide the best results. The maximum number of decoder iterations was set to 500 in case the PF-BP algorithm did not converge, and the scalar Lloyd-Max quantizer, trained using side information, is used for quantization. To keep the overall complexity low and for proof-of-concept, we use lowcomplexity regular LDPC codes with variable node degree 4 for SW coding [9]. More complex irregular codes would result in improved overall performance.

As a benchmark scheme, we use a DSC scheme that uses the same LDPC code and the same code length for compression, but exploits standard BP decoding without correlation tracking.

Figure 6 shows the obtained results as the required coding rate vs. MSE. Each sample is quantized using scalar quantization into either $q=4$ or $q=6$ bits, and SW coding is done bitplane-by-bitplane. Thus, $q$ different LDPC codes are used. The number of syndrome bits per bitplane/layer is determined heuristically to minimize the residual bit error rate. We initialise and maintain the correlation noise unchanged as white Gaussian with variance $\sigma^{2}=0.01$.

It can be seen from the figure that the proposed PF scheme outperforms the benchmark scheme. This is despite the fact that the correlation noise statistics do not vary over time. As expected, at low rates, it is better to use $q=4$ quantization levels, whereas at the high rates, $q=6$ provides slightly better performance. At 1 bits/sample and $q=4$ the benchmark scheme reaches the performance of the PF scheme and the remaining noise is only the quantization noise. For $q=6$, the proposed scheme shows error-free SW decoding performance at rate 2 bits/samples, while the benchmark scheme only at 4 bits/sample. The figure also shows the theoretical limit derived in the previous section. Note that the additional estimation step is applied after SW decoding. Since the resulting estimation gain diminishes as quantization step size decreases (see [7], [16]) a large gap to the bound occurs at higher rates.

The next figure shows results when the noise is white Gaussian with variance that follows Gaussian distribution and has mean of $\sigma^{2}=0.01$. That is, the variance slowly varies over time. As expected, the proposed PF-based scheme significantly outperforms the benchmark scheme. Thus, the proposed PFbased scheme successfully tracks the changes in the correlation and "adapts" BP decoding. Note that DSC theorems only hold when the source statistics is assumed to be constant. Therefore, no theoretical bound is shown in Figure 7. One can see that the trends are very similar to the previous example. A small gap between $q=4$ and $q=6 \mathrm{PF}$ curves for high rates is due to a lower quantization loss when 6 bits are used for quantization.

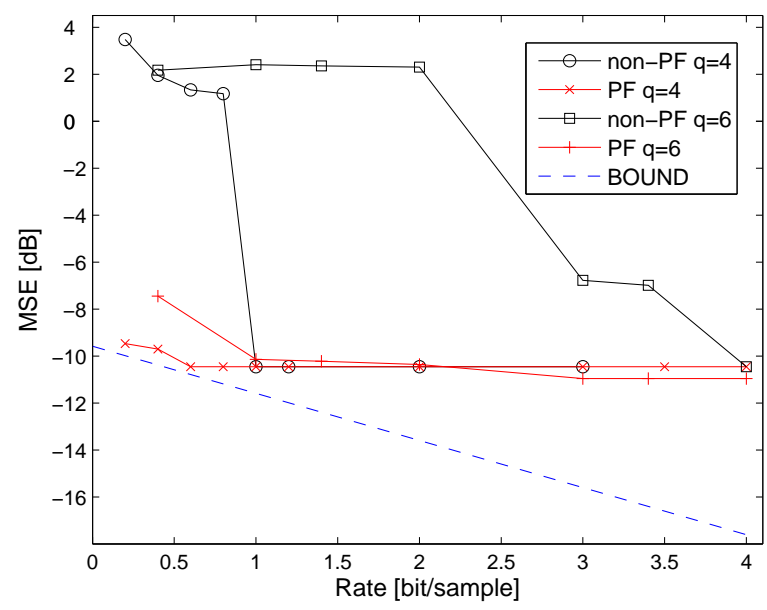

Fig. 6. Code rate vs. MSE for $q=4$ and $q=6$ and $\sigma^{2}=0.01$.

It can be seen from the two figures that significant savings in communications bandwidth can be achieved using the proposed solution.Indeed, assuming sampling rate of $f_{s}$ samples/sec, the proposed scheme at $q=4$ requires roughly for $0.5 f_{s} \mathrm{bit} / \mathrm{sec}$ and $2.5 f_{s}$ bit/sec less bandwidth than non-PF scheme, for $q=4$ and $q=6$, respectively.

\section{CONClusion}

In this paper we proposed a scheme for compressing wind speed measurements in a wind farm. Wind speed between wind farms provides important information necessary to estimate the amount of energy that can potentially be generated by the wind farm. The proposed solution exploits both temporal and spatial correlation between the wind farms' readings via distributed source coding. Moreover, the nonstationarity of the correlation model is taken care of with the particle-filtering based belief propagation at the decoder. The resulting scheme has low encoding complexity while being able to exploit 


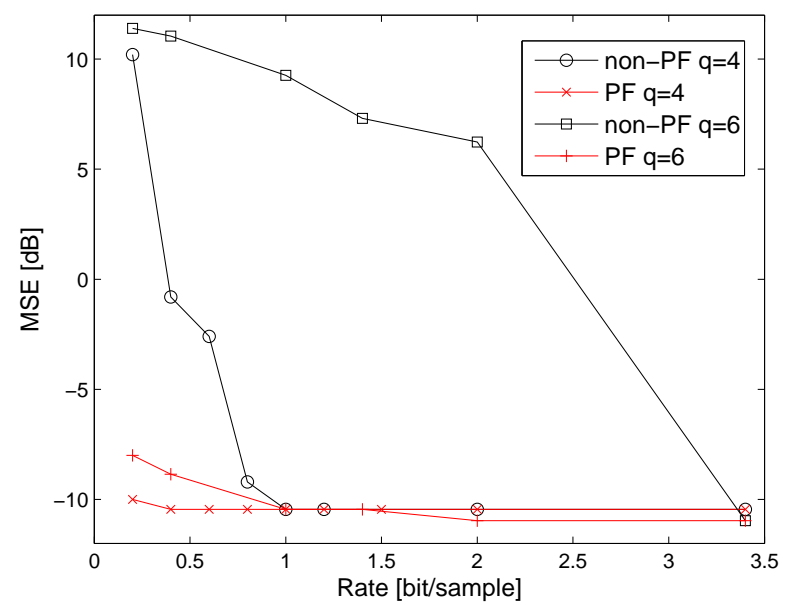

Fig. 7. Code rate vs. MSE for $q=4$ and $q=6$ and varying correlation noise.

correlation between the wind farms and dynamically track the changes in correlation noise. Simulation results using realistic models show impressive performance improvements compared to the scheme that does not track correlation.

\section{REFERENCES}

[1] K.R.W. Bell, D.C. Hill, D. McMillan, F. Li, R.W. Dunn, D.G. Infield, and G.W. Ault, "Spatio-temporal correlations of available wind power and impact on transmission power flows," 2010 CIGRE Techical Exibition, Paris, France, Aug. 2010.

[2] D.C. Hill, D. McMillan, K.R.W. Bell, and D. Infield, "Application of auto-regressive models to U.K. wind speed data for power system impact studies," IEEE Trans. Suistainable Energy, vol. 3, pp.134-141, Jan. 2012.

[3] D. Slepian and J.K. Wolf, "Noiseless coding of correlated information sources," IEEE Trans. Inform. Theory, vol. IT-19, pp. 471-480, July 1973.

[4] A.D. Wyner and J. Ziv, "The rate-distortion function for source coding with side information at the decoder," IEEE Trans. Inform. Theory, vol. IT22, pp. 1-10, Jan. 1976.

[5] T. Berger, "Multiterminal source coding," The Information Theory Approach to Communications, G. Longo, Ed., New York: Springer-Verlag, 1977.

[6] S.S. Pradhan and K. Ramchandran, "Distributed source coding using syndromes (DISCUS): design and construction," Proc. Data Compression Conference (DCC), Snowbird, UT, March 1999.

[7] Z. Xiong, A. Liveris, and S. Cheng, "Distributed source coding for sensor networks," IEEE Signal Processing Magazine, vol. 21, pp. 80-94, Sept. 2004

[8] V. Stanković, L. Stanković, and S. Cheng, "Distributed source coding: Theory and applications," in Proc. Eusipco 2010 European Signal Proc. Conf., Aalborg, Denmark, Aug. 2010.

[9] S. Wang, L. Cui, and S. Cheng, "Adaptive Wyner-Ziv decoding using particle-based belief propagation," in Proc. IEEE GlobeCom 2010, Miami, FL, Dec. 2010.

[10] L. Stanković, V. Stanković, S. Wang, and S. Cheng, "Correlation estimation with Particle-based Belief Propagation for distributed video coding," Proc. ICASSP-11 IEEE Intl. Conf. Acoustic, Speech, and Signal Proc., Prague, Czech Republic, May 2011.

[11] B. Girod, A. Aaron, S. Rane, and D. Rebollo-Monedero, "Distributed video coding," Proc. of the IEEE, vol. 93, pp. 71-83, Jan. 2005.

[12] R. Puri and K. Ramchandran, "PRISM: A new robust video coding architecture based on distributed compression principles," 40th Allerton Conf. Communication, Control and Computing, Allerton, IL, Oct. 2002.

[13] S. Wang, L. Cui, S. Cheng, L. Stanković, and V. Stanković, "Onboard low-complexity compression of solar images", ICIP-2011 IEEE Intl. Conf. Image Proc., Brussels, Belgium, Sept. 2011 (invited).

[14] V. Stanković, A. Liveris, Z. Xiong, and C. Georghiades, "On code design for the Slepian-Wolf problem and lossless multiterminal networks," IEEE Trans. Inform. Theory, vol. 52, pp. 1495-1507, April 2006.
[15] R. Zamir, "The rate loss in the Wyner-Ziv problem", IEEE Trans. Inform. Theory, pp. 2073-2084, Nov. 1996.

[16] Y. Yang, V. Stanković, Z. Xiong, and W. Zhao, "On multiterminal source code design," IEEE Trans. Inform. Theory, vol. 54, pp. 2278-2302, May 2008 .

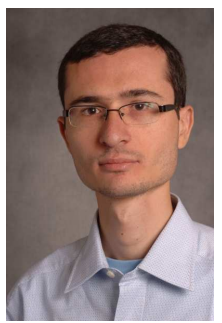

Vladimir Stankovic (M'03-SM'10) received the Dr.-Ing. (Ph.D.) degree from the University of Leipzig, Leipzig, Germany in 2003.

From 2003 to 2006, he was with Texas A\&M University, College Station. From 2006 to 2007 he was with Lancaster University. Since 2007, he has been with the Dept. Electronic and Electrical Engineering at University of Strathclyde, Glasgow, where he is currently a Senior Lecturer.

He has been an IET TPN Vision and Imaging Executive Team member and a member of the Technical Committee on the IEEE Communications Review Board (2010-2011). He was an Associate Editor of IEEE Communications Letters (2006-2012). $\mathrm{He}$ is currently an Editor of IEEE Transactions on Communications and a Technical Program Committee co-chair of Eusipco-2012.

His research interest include smart home communications, image/signal processing, and source/channel/network coding.

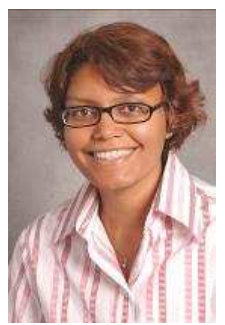

Lina Stankovic (M'03) received her BEng Hons and $\mathrm{PhD}$ degrees in Electronic Communications from Lancaster University, UK in 1999 and 2003, respectively.

She is a Lecturer at the University of Strathclyde since Oct 2007, Area editor of the Int. J. Communications (Elsevier), and UK Research Council peerreview College Member. She has published over 70 research papers in peer-reviewed international journals and conference proceedings as well as 4 book chapters.

Her research is mostly industrially driven with interests in coding, wireless communications, data analytics and decision support.

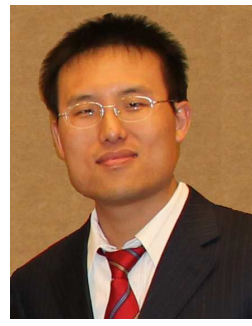

Shuang Wang received the B.S. degree in Applied Physics and M.S. degree in Biomedical Engineering from the Dalian University of Technology, China, and M.S. degree in Electrical and Computer Engineering from the University of Oklahoma, USA, in 2009.

He worked in Soft Imaging LLC, Houston, TX, in the areas of digital watermarking for Biomedical Image and GPU based next generation parallel genome sequencing algorithm during the summers of 2008 and 2009. He has one patent submission in the area of channel coding. He is awarded the Robert Hughes Centennial Fellowship since Fall 2009. Currently, he is working as a graduate assistant and a Ph.D. candidate under Dr. Samuel Cheng in OU-Tulsa campus.

His research interests include information theory, image/signal processing, and pattern recognition. 


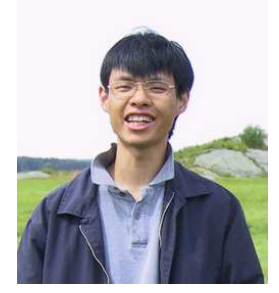

Samuel Cheng received the B.S. degree in Electrical and Electronic Engineering from the University of Hong Kong, and the M.Phil. degree in Physics and the M.S. degree in Electrical Engineering from Hong Kong University of Science and Technology and the University of Hawaii, Honolulu, respectively. He received the Ph.D. degree in Electrical Engineering from Texas A\&M University in 2004

He worked in Microsoft Asia, China, and Panasonic Technologies Company, New Jersey, during the summers of 2000 and 2001. In 2004, he joined Advanced Digital Imaging Research, a research company based near Houston, Texas, as a Research Engineer. Since 2006, he joined the School of Electrical and Computer Engineering at the University of Oklahoma and is currently an assistant professor.

His research interests include information theory, image/signal processing, and pattern recognition. 